\title{
MicroRNA 150-5p Improves Risk Classification for Mortality within 90 Days after Acute Ischemic Stroke
}

\author{
Natalie Scherrer, ${ }^{\mathrm{a}}$ Francois Fays, ${ }^{\mathrm{b}}$ Beat Mueller, ${ }^{\mathrm{c}}$ Andreas Luft, ${ }^{\mathrm{d}}$ Felix Fluri, ${ }^{\mathrm{c}}$ Mirjam Christ-Crain, ${ }^{\mathrm{e}}$ \\ Yvan Devaux, ${ }^{\mathrm{f}}$ Mira Katan ${ }^{\mathrm{a}}$ \\ aDepartment of Neurology, University Hospital of Zurich, Zurich, Switzerland \\ ${ }^{b}$ Competence Center in Methodology and Statistics, Luxembourg Institute of Health, Strassen, Luxembourg \\ 'Medical University Clinic, Cantonal Hospital Aarau, Aarau, Switzerland \\ ${ }^{d}$ Department of Neurology, University Hospital of Würzburg, Würzburg, Germany \\ 'Department of Endocrinology, University Hospital of Basel, Basel, Switzerland \\ ${ }^{f}$ Cardiovascular Research Unit, Luxembourg Institute of Health, Strassen, Luxembourg
}

Background and Purpose Micro ribonucleic acid-150-5p (miR-150-5p) regulates proinflammatory cytokines as well as vessel integrity. We evaluated the incremental prognostic value of logarithm $(\mathrm{log})$ of miR-150-5p plasma levels after ischemic stroke.

Methods In a prospective cohort study, levels of miR-150-5p were measured within 72 hours of symptom onset in 329 ischemic stroke patients. The outcome measures were unfavorable functional outcome (assessed by the modified Rankin Scale score $>2$ ) and mortality within 90 days. Logistic regression and Cox proportional hazards models were fitted to estimate odds ratio $(\mathrm{OR})$, respectively hazard ratio (HR) and 95\% confidence interval (CI) for the association between $\log -m i R-150-5 p$ and the outcome measures. The discriminatory accuracy was assessed with the area under the receiver-operating-characteristic curve (AUC) and the incremental prognostic value was estimated with the net reclassification index.

Results After adjusting for demographic and vascular risk factors, lower log-miR-150-5p levels were independently associated with mortality ( $\mathrm{HR} 0.21[95 \% \mathrm{Cl}, 0.08-0.51], P=0.001)$ but not functional outcome (OR 1.10 [95\% Cl, 0.54-2.25], $P=0.79$ ). Adding log-miR-150-5p improved the discriminatory accuracy of the best multivariate model to predict mortality from an AUC of 0.91 (95\% Cl, 0.88-0.95) to $0.92(95 \% \mathrm{Cl}, 0.88-0.96$ Likelihood-ratio test- $P<0.001)$, and resulted in a net reclassification index of $37.3 \%(95 \% \mathrm{Cl}, 0.28-0.52)$.

Conclusions In patients with ischemic stroke, log-miR-150-5p is a novel prognostic biomarker, highly associated with mortality within 90 days, improving risk classification beyond traditional risk factors.
Correspondence: Mira Katan Department of Neurology, University Hospital of Zurich, Frauenklinikstrasse 26, 8091 Zurich, Switzerland

Tel: $+41(0) 442554732$

Fax: +41 (0)44 2554380 E-mail: mira.katan@usz.ch

Received: March 6, 2017

Revised: July 12, 2017

Accepted: July 12, 2017

This work was supported by the Swiss National Science Foundation (PZOOP3_142422 [MK]), the Fondation Leducq ([MK]) and the Ministry of Higher Education and Research of Luxembourg [YD]. All supporting entities had no role in the collection, management, analysis, and interpretation of the data; or the preparation, or approval of the manuscript.

The authors have no financial conflicts of interest.

Keywords MicroRNAs; Prognosis; Stroke; Biomarkers; Mortality; Ischemic stroke

\section{Introduction}

Knowledge of the important factors that affect prognosis of stroke is necessary to accurately predict outcome of patients, to provide a rational approach to patient management, and to understand the disease development.

Some clinical scores have been proposed to predict functional outcome ${ }^{1}$ and mortality, ${ }^{2}$ however there is room for 
improvement. Rapidly measurable biomarkers, which are associated with disease development, functional outcome and mortality, may improve the prognostic accuracy of traditional risk factors. However, to be clinically useful in the setting of acute stroke, blood biomarkers have to add prognostic information to already established risk factors such as stroke severity and age. ${ }^{3}$ The search for such biomarkers has become increasingly popular ${ }^{4}$ however only in very few cases ${ }^{5}$ they showed incremental predictive value.

MiRNAs are short (approximately 22 nucleotides) noncoding RNA molecules involved in post-transcriptional gene regulation. ${ }^{6}$ Their presence and stability in the bloodstream led to the hypothesis that they might serve as biomarkers. ${ }^{7}$ In cardiovascular diseases, circulating levels of micro ribonucleic acid-150-5p (miR-150-5p) were found to be associated with outcome after acute myocardial infarction. ${ }^{8}$ Expressed mainly by monocytes, miR-150-5p acts as both an endocrine factor leading to anti-inflammatory effects ${ }^{9}$ and a paracrine factor leading to vascular endothelial cell migration. ${ }^{10}$ In view to our study, other authors described that the expression of miR-150 was decreased in brain and serum of rats subjected to cerebral ischemia. ${ }^{11}$ Due to its ability to regulate vessel integrity and pro-inflammatory cytokines secretion, mediators of cellular communication in the ischemic brain, we hypothesized that miR-150-5p may inform about the prognosis of patients with acute ischemic stroke. In this study, we measured the plasma levels of miR150-5p in a cohort of 329 patients with acute ischemic stroke and we determined their incremental predictive value over traditional demographic and vascular risk factors.

\section{Methods}

\section{Study design and setting}

The design of the prospective cohort used in the present study (https://www.clinicaltrials.gov/; NCT00390962) has been described in detail elsewhere. ${ }^{12} \mathrm{~A}$ flow-chart of the present study is provided in Figure 1. Briefly, 605 patients with suspected ischemic cerebrovascular events presenting at the emergency department between October 2006 and October 2007 were screened. Of these, 362 patients had an ischemic stroke according to the World Health Organization (WHO) criteria, 359 completed follow up (99.2\%), and 329 had plasma samples available for the analysis of logarithm (log)-miR-150-5p. Initial ischemic stroke was defined upon to the WHO criteria as an acute focal neurological deficit lasting longer than 24 hours $^{13}$ with no sign of acute intracranial bleeding on cerebral imaging. Missing informed consent or any diagnosis different from

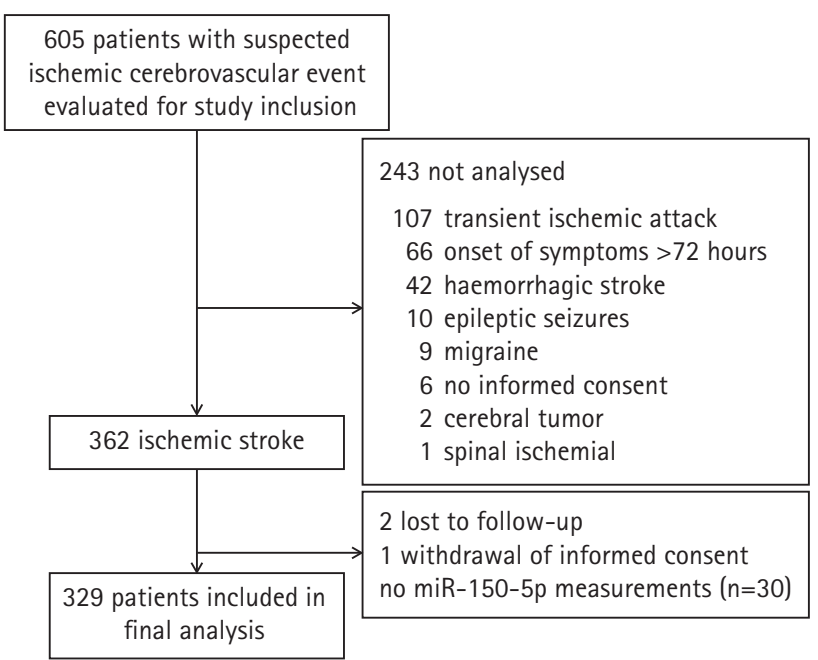

Figure 1. Flowchart of patient's enrollment and follow up. From 605 screened patients, ischemic stroke was diagnosed in 362 patients. 359 patients completed follow-up and were finally included in the analysis. Of these, 30 patients were excluded from the analysis because of missing plasma samples for miR-150-5p determination.

ischemic stroke (i.e., stroke mimics) were exclusion criteria. The study was approved by the Ethics Committee of Basel, Switzerland and was conducted according to the principles expressed in the Declaration of Helsinki. All patients or the patients' next of kin provided written informed consent for the collection of data, blood samples, and subsequent analyses.

\section{Clinical baseline variables}

The following data were collected with a standardized bed-side interview and complete chart review on admission: vital signs, co-morbidities as assessed by the Charlson Comorbidity Index adjusted for stroke (such as age, gender, smoking habits, history of hypercholesterolemia, hypertension, diabetes mellitus, previous stroke, positive family history for stroke and history of coronary heart disease), medication at the time of index ischemic stroke and cardiovascular risk factors (i.e., age, sex, smoking habits, history of hypercholesterolemia, hypertension, diabetes mellitus, previous stroke, positive family history for myocardial infarction, stroke and history of coronary heart disease). Stroke physicians prospectively recorded the National Institute of Health Stroke Scale (NIHSS) score ${ }^{14}$ upon admission. Stroke etiology was determined according to the criteria of the Trial of ORG 10172 in Acute Stroke Treatment (TOAST) criteria, ${ }^{15}$ which distinguishes large-artery atherosclerosis, cardioembolism, small-artery occlusion, other etiology and undetermined etiology.

\section{Blood sampling}

In all patients the routine admission laboratory tests consisted 
of at least the following parameters, $\mathrm{C}$ - reactive protein (CRP), creatinine, glucose, cholesterol, triglycerides and white blood cells. In addition, for miR-150-5p assessment, plasma samples were collected in EDTA tubes on admission within 72 hours from symptom onset. These tubes were all centrifuged within 5 minutes, aliquoted and frozen at $-70^{\circ} \mathrm{C}$ within 20 minutes from blood draw, for future analyses.

\section{MiR-150-5p assessment}

Circulating levels of miR-150-5p were measured blinded to clinical outcomes, as described in details elsewhere. ${ }^{16}$ Briefly, total RNA was extracted from plasma samples with the mirVana PARIS kit (Ambion, Applied Biosystem, Lennik, Belgium). The elution volume was $50 \mu \mathrm{L}$, of which $12 \mu \mathrm{L}$ were used as input for Reverse-transcription. No enrichment for small RNAs was performed. The synthetic C. elegans miR-39 (Qiagen, Venlo, the Netherlands), which is devoid of sequence homology to human miRNAs, was added to plasma samples after lysis to correct for extraction efficiency. Potential DNA contamination was eliminated using DNase (Qiagen). RNA was reverse transcribed with the miScript reverse transcription kit (Qiagen) and the resulting cDNA was diluted 10-fold before quantitative polymerase chain reaction (PCR) with the miScript SYBRgreen PCR kit (Qiagen). MiR-150-5p-specific miScript primers were obtained from Qiagen. An inter-run calibrator was included in each PCR plate to correct for inter-run technical variations.

Expression levels were normalized with cel-miR-39 according to the formula (2exp[Ct cel-miR-39/Ct miR-150-5p]), and are expressed as number of copies of miR-150-5p per $\mu \mathrm{L}$ of plasma, as previously described for other miRNAs. ${ }^{17}$

\section{Outcome measures}

Trained stroke physicians and study nurses assessed outcome three months after the acute stroke with a structured followup telephone interview. They were blinded to log-miR-150-5p levels and baseline clinical variables.

The outcome measures were unfavorable functional outcome defined as a modified Rankin Scale (mRS) score of $>2$ points and mortality. As secondary outcome measure we used the mRS as ordinal variable (ordinal scale from 0 to 6 points). All outcome measures were assessed at 90 days after the index stroke.

\section{Neuroimaging}

Ischemic stroke was a clinical diagnosis according to the WHO criteria, but computed tomography was performed in all patients on admission mainly to exclude intracranial hemorrhage. Additionally, magnetic resonance imaging with diffusionweighted imaging was performed in 188 patients (57.1\%). Dif- fusion-weighted imaging lesion volumes were measured by the consensus of two experienced raters unaware of the clinical and laboratory findings. A semi-quantitative method validated for ischemic stroke lesions was used to calculate the lesion size. ${ }^{18}$ Lesions were ranked into three size classes to represent typical stroke patterns: (1) small lesion with a volume of $<10$ $\mathrm{mm}^{3}$, (2) medium lesion of $10-100 \mathrm{~mm}^{3}$, (3) large lesion with a volume of more than $100 \mathrm{~mm}^{3} .^{12}$

\section{Statistical analysis}

Statistical analysis was performed for both outcome measures separately. Discrete variables are expressed as frequency (percentage), continuous variables as medians with interquartile ranges (IQR). The distribution of raw biomarker data was skewed. After log transformation with a base of 10, the distribution of the biomarker data approximated a normal distribution. Thus one-unit increase of log-miR-150-5p corresponds to a tenfold increase in miR-150-5p levels.

Two group comparisons for categorical baseline measurements were performed by Fisher's exact test and for continuous, not normally distributed baseline data, by the MannWhitney $U$ test. For simple survival analysis, we stratified patients according to log-miR-150-5p quartiles in Kaplan Meier curves and compared the groups by mean of the log-rank test.

To assess the independent association of log-miR-150-5p levels with our outcome measures, we computed a multivariate binary and ordinal logistic and Cox regression model. The final multivariate models included variables significantly associated with an unfavorable outcome or mortality in the univariate analyses (for functional outcome: log-miR-150-5p, CRP, sex, atrial fibrillation, Charlson comorbidity index, age, NIHSS, total anterior circulation stroke, posterior circulation stroke; for mortality: log-miR-150-5p, CRP, atrial fibrillation, age, NIHSS, total anterior circulation stroke). We report odds ratio (OR) and hazard ratio (HR) along with 95\% confidence interval (Cl) as measure of association and uncertainty, respectively.

To account for missing values, we performed multiple imputations, which have been recently proposed to be the best approach for modeling the effects of missing data. ${ }^{19}$

The variable with the most missing values (i.e., CRP levels) included in the multivariate model was absent in $16.7 \%$ of all included patients. All variables included in the regression analysis were used in the imputation process as recommended. ${ }^{20}$

No relevant differences between the results of the model with and without the multiple imputations were observed (neither regarding the magnitude nor the significance of associations), thus, in order to improve the power of the analysis, we calculated all further steps (see next paragraph) based on 
the multiple imputation models.

The discriminatory value of log-miR-150-5p was assessed with the area under the receiver-operating-characteristic curve (AUC). For the risk model of comparison, we employed the multivariate models described above. As recommended in the current statistical literature, ${ }_{1}^{21}$ we calculated continuous (category-free) net reclassification index (NRI) since our multivariate models of comparisons have no validated risk categories.

Statistics were calculated using Stata Statistical Software ver. 13.1 (StataCorp LLC, College Station, TX, USA). Testing was two-sided and $P$ values less than 0.05 were considered to indicate statistical significance.

\section{Results}

\section{Study population}

From 605 screened patients, ischemic stroke was diagnosed in 362 patients, and 359 completed follow-up. ${ }^{12} 329$ patients had blood samples available for the measurement of miR-150-5p (Figure 1). To evaluate the potential time dependence of the time of blood draw within the 72 hours after symptom onset and the log-miR-150-5p levels we conducted a Kruskal-wallis test which showed no statistically significant correlation $(P=0.62)$. To assess the effect of "time to blood draw" on the association of miR150-5p with the outcome variable mortality, we performed a bivariate cox regression analysis with miR$150-5 p$ as main predictor adjusting for the variable "time to blood draw." MiR150-5p remained independently associated with mortality with a HR of $0.17(95 \% \mathrm{Cl}, 0.08-0.36, P<0.00)$.

The distribution of demographic and vascular risk factors was similar between the original cohort of 359 patients (details provided in an earlier report ${ }^{5}$ ) and the 329 patients with available blood samples used in the present study.

As shown in Table 1, the median age of the present cohort of 329 patients was 75 years (IOR 65-82), identical to the original cohort of 359 patients (75 years [IOR 63-83]). 41\% of the 329 patients were women (original cohort 41\%). The most common cardiovascular risk factor was arterial hypertension, which was present in 76\% of patients (original cohort 77\%). At admission, the median NIHSS score was 5 (IOR 2-10) (original cohort 5 [IQR 2-10]), and the median log-miR-150-5p concentration was 1,357.1 copies per $\mu \mathrm{L}$ of plasma (IQR 755.8-2,869.3).

\section{Log-miR-150-5p and functional outcome at 90 days}

A total of $134(40.7 \%)$ patients had an unfavorable outcome after 90 days. Patients with unfavorable outcome were older, suffered more often from arterial hypertension and had a higher NIHSS score on admission (Table 2). The median logmiR-150-5p concentration was lower in patients who had an unfavorable outcome at 90 days as compared to patients with a favorable outcome (Table 2). In the univariate model, lower log-miR-150-5p levels were associated with unfavorable functional outcome with an OR of $0.47(95 \% \mathrm{Cl}, 0.28-0.78$, $P<0.01)$. However, in the multivariate analysis, log-miR-150-5p was no longer associated with unfavorable functional outcome (OR 1.10 [95\% Cl, 0.54-2.55], $P=0.79$ ) (Table 3). The same was observed when using standardized micro RNA levels as odds ratio per 1-SD instead of log levels (OR 1.21 [95\% Cl, 0.871.68], $P=0.27$ ). When analyzing functional outcome as ordinal scale (mRS 1-6 points) we also did not find an independent association of log-miR-150-5p ( $P=0.19)$. The multivariate logistic model was well calibrated as assessed by the Hosmer and Lemeshow goodness-of-fit test $(P=0.27)$.

\section{Log-miR-150-5p and mortality within 90 days}

A total of 39 (11.9\%) patients died within 90 days after stroke. The median log-miR-150-5p concentration was lower in patients who died within 90 days as compared to survivors (Table 1). Overall, Kaplan-Meier survival curves of patients stratified per log-miR-150-5p quartiles differed $(P<0.001$, log-rank test) (Figure 2). In the univariate Cox model, log-miR-150-5p levels were associated with mortality with a $\mathrm{HR}$ of $0.18(95 \% \mathrm{Cl}$, 0.08-0.39; $P<0.001)$.

In the multivariate Cox model, lower log-miR-150-5p concentrations independently predicted mortality ( $\mathrm{HR} 0.21[95 \% \mathrm{Cl}$, 0.08-0.51], $P=0.001)$. The same association was found after standardization of the log-units of miR-150-5p for mortality (HR 0.49 [95\% Cl, 0.33-0.75], $P=0.001$ ). The multivariate Cox model was well enough calibrated as assessed by the Groennesby and Borgan test $(P=0.06)$. Also miR-150-5p remained independently associated with mortality after adjusting for copeptina well validated prognostic stroke biomarker $5(0.21[95 \% \mathrm{Cl}$, 0.07-0.63], $P<0.01$ ). Log-miR150 and the risk factors which were statistically significant in the univariate analyses for mortality are shown in the scatter and box plots (Supplementary Figure 1).

Adding log-miR-150-5p improved the discriminatory accuracy of the NIHSS alone (AUC from $0.84[95 \% \mathrm{Cl}, 0.83-0.86]$ to 0.86 [95\% Cl, 0.84-0.87], Likelihood-ratio test $P<0.001$ ) (Table 4). Moreover log-miR-150-5p improved the discriminatory accuracy of the whole multivariate model (AUC from 0.91 [95\% $\mathrm{Cl}, 0.88-0.95]$ to 0.92 [95\% $\mathrm{Cl}, 0.88-0.96]$, Likelihood-ratio test $P<0.001$ ) (Table 4). These results were further strengthened by a NRI of $37.3 \%(95 \% \mathrm{Cl}, 0.28-0.52)$ and the pseudo R-squared test whose difference of 135.416 in Bayesian-Information-Criterion provides very strong support for our current model. 
Table 1. Baseline characteristics of all patients as well as stratified by mortality

\begin{tabular}{|c|c|c|c|c|}
\hline & All patients $(n=329)$ & No death $(n=290)$ & Death $(n=39)$ & $P$ \\
\hline \multicolumn{5}{|l|}{ Demographic data } \\
\hline Age (years) & $75(65-82)$ & $74(62-82)$ & $82(78-87)$ & $<0.001$ \\
\hline Women & $136(41)$ & $119(41)$ & $17(44)$ & 0.86 \\
\hline \multicolumn{5}{|l|}{ Medical history } \\
\hline Hypertension & $251(76)$ & $219(76)$ & $32(82)$ & 0.43 \\
\hline Atrial fibrillation & $66(20)$ & $49(17)$ & $17(44)$ & $<0.001$ \\
\hline Current smoking & $116(35)$ & $105(36)$ & $11(28)$ & 0.38 \\
\hline Diabetes mellitus & $66(20)$ & $57(20)$ & $9(23)$ & 0.67 \\
\hline Coronary heart disease & $84(26)$ & $69(24)$ & $15(38)$ & 0.08 \\
\hline Dyslipidemia & $90(27)$ & $79(27)$ & $11(28)$ & 0.85 \\
\hline Previous cerebrovascular event & $80(24)$ & $72(25)$ & $8(21)$ & 0.69 \\
\hline Charlson comorbidity Index & $1(0-2)$ & $1(0-2)$ & $1(0-2)$ & 0.04 \\
\hline Infections at admission* & $21(6.6)$ & $16(5.7)$ & $5(13.5)$ & 0.08 \\
\hline \multicolumn{5}{|l|}{ Clinical data } \\
\hline NIHSS at admission (points) & $5(2-10)$ & $4(2-8)$ & $15(8-25)$ & $<0.001$ \\
\hline \multicolumn{5}{|l|}{ Stroke syndrome and causative factors } \\
\hline Total anterior circulation stroke & $35(11)$ & $21(7)$ & $14(36)$ & $<0.001$ \\
\hline Partial anterior circulation stroke & $150(46)$ & $131(45)$ & $19(49)$ & 0.73 \\
\hline Lacunar anterior circulation stroke & $67(20)$ & $63(22)$ & $4(10)$ & 0.14 \\
\hline Posterior circulation stroke & $89(27)$ & $82(28)$ & $7(18)$ & 0.25 \\
\hline \multicolumn{5}{|l|}{ Laboratory values } \\
\hline Log-miR-150-5p $p^{+}$ & 1,357.1 (755.8-2,869.3) & $1,452.9(845.3-3,116.4)$ & $777.3(577.7-1,326.4)$ & $<0.001$ \\
\hline Glucose (mmol/L) & $6.1(5.5-7.4)$ & $6.0(5.4-7.4)$ & $6.3(6.0-7.5)$ & 0.25 \\
\hline $\mathrm{CRP}(\mathrm{mg} / \mathrm{L})^{\ddagger}$ & $3.5(3.0-9.8)$ & $3.2(3.0-8.6)$ & $14.0(3.9-43.8)$ & $<0.001$ \\
\hline White blood cells per $\mu \mathrm{L}$ & $8.3(6.6-9.9)$ & $8.2(6.6-9.7)$ & $9.3(6.5-12.1)$ & 0.12 \\
\hline Temperature $\left({ }^{\circ} \mathrm{C}\right)$ & $37.0(36.5-37.5)$ & $37.0(36.5-37.4)$ & $37.2(36.4-37.6)$ & 0.79 \\
\hline Lesion size on $\mathrm{MR}$, DW/ ${ }^{\S}$ & $1.7(0.2-19.4)$ & $1.5(0.2-16.4)$ & $40.0(3.4-146.8)$ & 0.001 \\
\hline Thrombolysis & $63(19.8)$ & $54(19.1)$ & $38(20.0)$ & 0.26 \\
\hline \multicolumn{5}{|l|}{ TOAST ${ }^{15}$ subtype } \\
\hline Large-vessel disease & $61(19)$ & $56(19)$ & $5(13)$ & 0.39 \\
\hline Cardioembolic & $121(37)$ & $103(36)$ & $18(46)$ & 0.22 \\
\hline Small-artery disease & $50(15)$ & $49(17)$ & $1(3)$ & 0.02 \\
\hline Other known & $15(5)$ & $14(5)$ & $1(3)$ & 1.00 \\
\hline Undetermined & $82(25)$ & $68(24)$ & $14(36)$ & 0.11 \\
\hline \multicolumn{5}{|l|}{ Time from symptom onset till blood draw } \\
\hline $0-3$ hours & $71(21.9)$ & $59(20.7)$ & $12(30.8)$ & 0.15 \\
\hline $3-12$ hours & $169(52.2)$ & $151(52.98)$ & $18(46.2)$ & 0.50 \\
\hline $12-24$ hours & $48(14.8)$ & 45 (15.8) & $3(7.7)$ & 0.23 \\
\hline $24-72$ hours & $36(11.11)$ & $30(10.5)$ & $6(15.4)$ & 0.41 \\
\hline
\end{tabular}

Values are presented as median (meaning range between the first and third quartile) or number (\%).

NIHSS, National Institute of Health Stroke Scale; CRP, C-reactive protein; MR, magnetic resonance; DWI, diffusion weighted imaging; TOAST, Trial of ORG 10172 in Acute Stroke Treatment.

${ }^{*}$ All types of infections including pneumonia on admission or history within the last week; ${ }^{+}$Log 10 -transformed values were used, thus one-unit increase corresponds to a tenfold increase in normalized copy numbers per $\mu \mathrm{L}$ of plasma. See the "Statistical Analysis" section for details; ${ }^{*} \mathrm{CRP}$ had missing values (missing in $16.7 \%$ of patients); ${ }^{5}$ Volume in milliliters. Refers to patients where information on diffusion-weighted imaging lesion was present ( $\left.n=188\right)$. 
Table 2. Baseline characteristics of all patients, stratified by functional outcome

\begin{tabular}{|c|c|c|c|c|}
\hline & All patients $(n=329)$ & Favorable outcome $(n=195)$ & Unfavorable outcome $(n=134)$ & $P$ \\
\hline \multicolumn{5}{|l|}{ Demographic data } \\
\hline Age (years) & $75(65-82)$ & $68.6(20.4-94.2)$ & $78.0(44.6-96.6)$ & $<0.001$ \\
\hline Women & $136(41)$ & $69(35.4)$ & $67(50.0)$ & $<0.01$ \\
\hline \multicolumn{5}{|l|}{ Medical history } \\
\hline Hypertension & $251(76)$ & $142(72.8)$ & 109 (81.3) & 0.09 \\
\hline Atrial fibrillation & $66(20)$ & 29 (14.9) & $37(27.6)$ & $<0.01$ \\
\hline Current smoking & $116(35)$ & $74(38.0)$ & $42(31.3)$ & 0.24 \\
\hline Diabetes mellitus & $66(20)$ & 38 (19.5) & $28(20.9)$ & 0.78 \\
\hline Coronary heart disease & $84(26)$ & $46(23.6)$ & $38(28.4)$ & 0.37 \\
\hline Dyslipidemia & $90(27)$ & $58(29.7)$ & $32(23.9)$ & 0.26 \\
\hline Previous cerebrovascular event & $80(24)$ & $45(23.1)$ & $35(26.1)$ & 0.60 \\
\hline Charlson comorbidity Index & $1(0-2)$ & $1(0-8)$ & $2(0-6)$ & $<0.001$ \\
\hline Infections at admission* & $21(6.6)$ & $9(4.7)$ & $12(9.5)$ & 0.11 \\
\hline \multicolumn{5}{|l|}{ Clinical data } \\
\hline NIHSS at admission (points) & $5(2-10)$ & $5(0-23)$ & $11(0-42)$ & $<0.001$ \\
\hline \multicolumn{5}{|l|}{ OCSP } \\
\hline TACS & $35(11)$ & $8(4.1)$ & $27(20.2)$ & $<0.001$ \\
\hline PACS & $150(46)$ & $87(44.6)$ & $63(47.0)$ & 0.74 \\
\hline LACS & $67(20)$ & $42(21.5)$ & $25(18.7)$ & 0.58 \\
\hline POCS & $89(27)$ & $61(31.3)$ & $28(20.9)$ & 0.04 \\
\hline \multicolumn{5}{|l|}{ Laboratory values } \\
\hline MiR-150-5p $p^{+}$ & 1,357.1 (755.8-2,869.3) & $4,103.5(185.4-180,811.3)$ & $1,980.3(40.6-11,540.5)$ & 0.01 \\
\hline Glucose (mmol/L) & $6.1(5.5-7.4)$ & $6.6(3.3-17.2)$ & $6.9(2.2-24.6)$ & 0.10 \\
\hline White blood cell per $\mu \mathrm{L}$ & $3.5(3.0-9.8)$ & $8.2(6.5-9.6)$ & $8.3(6.7-10.2)$ & 0.20 \\
\hline $\mathrm{CRP}(\mathrm{mg} / \mathrm{L})^{*}$ & $8.3(6.6-9.9)$ & $9.9(3.0-130.4)$ & $24.6(3.0-302.0)$ & $<0.001$ \\
\hline Temperature $\left({ }^{\circ} \mathrm{C}\right)$ & $37.0(36.5-37.5)$ & $37.0(35.0-38.3)$ & $37.0(35.3-39.9)$ & 0.56 \\
\hline Lesion size on $\mathrm{MR}, \mathrm{DW} \mathrm{I}^{\S}$ & $1.7(0.2-19.4)$ & $12.2(0.1-153.8)$ & $45.6(0.1-317.5)$ & $<0.001$ \\
\hline Thrombolyis & $63(19.8)$ & $38(20.0)$ & 25 (19.4) & 0.51 \\
\hline \multicolumn{5}{|l|}{ TOAST $^{15}$ subtype } \\
\hline Large-vessel disease & $61(19)$ & $36(18.5)$ & 25 (18.7) & 1.00 \\
\hline Cardioembolic & $121(37)$ & $70(35.9)$ & $51(38.1)$ & 0.73 \\
\hline Small-artery disease & $50(15)$ & 35 (18.0) & 15 (11.2) & 0.12 \\
\hline Other known & $15(5)$ & $10(5.1)$ & $5(3.7)$ & 0.60 \\
\hline Undetermined & $82(25)$ & $44(22.6)$ & $38(28.4)$ & 0.25 \\
\hline \multicolumn{5}{|c|}{ Time from symptom onset till blood draw } \\
\hline $0-3$ hours & $71(21.9)$ & $41(21.5)$ & $30(22.6)$ & 0.89 \\
\hline $3-12$ hours & $169(52.2)$ & $97(50.8)$ & $72(54.1)$ & 0.57 \\
\hline $12-24$ hours & $48(14.8)$ & $31(16.2)$ & $17(12.8)$ & 0.43 \\
\hline 24-72 hours & $36(11.11)$ & $22(11.5)$ & $14(10.5)$ & 0.86 \\
\hline
\end{tabular}

Values are presented as median (meaning range between the first and third quartile) or number (\%).

NIHSS, National Institute of Health Stroke Scale; OCSP, Oxfordshire Community Stroke Project; TACS, total anterior circulation syndrome; PACS, partial anterior circulation stroke; LACS, lacunar anterior circulation stroke; POCS, posterior circulation stroke; CRP, C-reactive protein; MR, magnetic resonance; DWI, diffusion weighted imaging; TOAST, Trial of ORG 10172 in Acute Stroke Treatment.

${ }^{*}$ All types of infections including pneumonia on admission or history within the last week; ${ }^{+}$Normalized copy number per $\mu \mathrm{L}$ of plasma; ${ }^{{ }^{*} \mathrm{CRP}}$ had missing values (missing in $16.7 \%$ of patients); ${ }^{5}$ Percentages refer to patients where information on DWI lesion was present $(n=188)$. 
Table 3. Multivariate logistic regression analysis for functional outcome and cox regression model for mortality

\begin{tabular}{|c|c|c|c|c|c|c|}
\hline \multirow{2}{*}{ Predictors } & \multicolumn{3}{|c|}{ Functional outcome } & \multicolumn{3}{|c|}{ Mortality } \\
\hline & $\mathrm{OR}$ & $95 \% \mathrm{Cl}$ & $P$ & $\mathrm{HR}$ & $95 \% \mathrm{Cl}$ & $P$ \\
\hline Log-miR-150-5p* & 1.10 & $0.54-2.25$ & 0.79 & 0.21 & $0.08-0.51$ & 0.001 \\
\hline $\mathrm{CRP}(\mathrm{mg} / \mathrm{L})$ & 1.01 & $1.00-1.02$ & 0.03 & 1.01 & $1.00-1.02$ & 0.001 \\
\hline Age (years) & 1.09 & $1.05-1.12$ & $<0.001$ & 1.05 & $1.01-1.10$ & 0.01 \\
\hline Sex & 1.34 & $0.75-2.39$ & 0.33 & & & \\
\hline NIHSS at admission (point) & 1.15 & $1.09-1.21$ & $<0.001$ & 1.10 & $1.06-1.14$ & $<0.001$ \\
\hline Charlson comorbidity index (point) & 1.45 & $1.19-1.76$ & $<0.001$ & & & \\
\hline Total anterior circulation stroke & 3.86 & $1.24-12.06$ & 0.02 & 2.51 & $1.09-5.78$ & 0.03 \\
\hline Atrial fibrillation & 0.52 & $0.25-1.10$ & 0.09 & 0.82 & $0.39-1.75$ & 0.62 \\
\hline Posterior circulation stroke & 0.79 & $0.41-1.52$ & 0.48 & & & \\
\hline
\end{tabular}

$\mathrm{OR}$, odds ratio; $\mathrm{Cl}$, confidence interval; $\mathrm{HR}$, hazard ratio; CRP, C-reactive protein; NIHSS, National Institute of Health Stroke Scale.

*Log10-transformed values of normalized copy numbers per $\mu \mathrm{L}$ of plasma were used. The variables which were statistically significant in the univariate analysis where included as predictors.

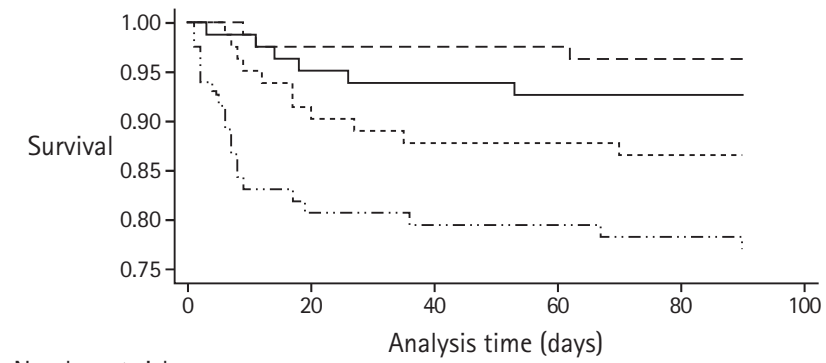

Number at risk

\begin{tabular}{|c|c|c|c|}
\hline $\mathrm{Q}=183$ & 67 & 66 & 66 \\
\hline $\mathrm{Q}=282$ & 75 & 72 & 72 \\
\hline $0=382$ & 78 & 77 & 76 \\
\hline \multirow[t]{2}{*}{$\mathrm{Q}=482$} & 80 & 80 & 80 \\
\hline & & $\begin{array}{l}0=1 \\
0=3\end{array}$ & -- \\
\hline
\end{tabular}

Figure 2. Kaplan-Meier survival estimates for patients stratified by logmiR-150-5p quartiles. The numbers of patients at risk are indicated at multiples of 20 days. Overall, Kaplan-Meier survival curves of patients stratified per log-miR-150-5p quartiles differed ( $P<0.001$, log-rank test). 0 means quartiles of log10-transformed values of miR-150-5p were used in these analyses. The $x$-axis is abridged and shows only the results of the 90 days after event.

\section{Subgroup of patients with available diffusion- weighted imaging measurements}

Admission magnetic resonance imaging with diffusion-weighted imaging was available in 188 acute stroke patients (57\%). Of these, 11 patients died (6\%) within 90 days after stroke. $68 \%$ of the patients had a small, $26 \%$ a medium and $6 \%$ a big lesion. In this subgroup of patients, log-miR-150-5p was still independently associated with mortality, even after adjustment for lesion size (HR 0.17 [95\% Cl, 0.04-0.77], $P=0.02$ ).
Table 4. AUC for selected predictors for mortality

\begin{tabular}{|c|c|c|c|}
\hline \multirow{2}{*}{ Predictors } & \multicolumn{3}{|c|}{ Mortality } \\
\hline & AUC & $95 \% \mathrm{Cl}$ & $P^{*}$ \\
\hline NIHSS & 0.84 & $0.83-0.86$ & $<0.001$ \\
\hline NIHSS+log miR-150-5p $p^{+}$ & 0.86 & $0.84-0.87$ & \\
\hline Model $1^{\ddagger}$ & 0.91 & $0.88-0.95$ & \\
\hline Model $1^{\dagger}+\log$ miR-150-5 $p^{\dagger}$ & 0.92 & $0.88-0.96$ & $<0.001$ \\
\hline
\end{tabular}

AUC, area under the receiver-operating-characteristic curve; $\mathrm{Cl}$, confidence interval; NIHSS, National Institute of Health Stroke Scale.

${ }^{*}$ To test the statistical significance of the comparisons of nested vs. whole models, the likelihood ratio test was used as recommended ${ }^{31}$ ${ }^{+} \log 10$-transformed values of normalized copy numbers per $\mu \mathrm{L}$ of plasma were used; ${ }^{\ddagger}$ Model 1: includes all variables except log miR-150-5p which were statistically significant in the univariate analyses for mortality (C-reactive protein, age, NIHSS at admission, total anterior circulation stroke, atrial fibrillation).

\section{Discussion}

Our main finding is that lower log-miR-150-5p plasma levels were independently associated with mortality but not functional outcome three months after ischemic stroke. Log-miR150-5p improved the discriminatory ability of the NIHSS and multivariate models as shown by an increase in the respective AUCs. Despite the rather modest increase of AUC, log-miR150-5p improved risk classification by $37.3 \%$ for mortality.

The pathophysiological mechanisms relating miR-150-5p with stroke are not fully unraveled. MiR-150-5p is found to be expressed in monocytes, ${ }_{1}^{9}$ endothelial progenitor cells, ${ }_{1}^{22}$ platelets, ${ }^{23}$ and human coronary artery endothelial cells. ${ }^{24}$ The influence of miR-150-5p was described in the context of cell proliferation, migration, and cell differentiation. ${ }^{25}$ Several mRNA targets of miR-150 have been identified and validated, includ- 
ing $\mathrm{CXCR} 4_{1}^{9,22}$ thereby possibly regulating inflammation which can affect the prognosis after stroke. Mobilization and migration of endothelial progenitor cells are mainly controlled by stromal cell-derived factor $1 \alpha$ (SDF-1 $\alpha$ ) and its receptor CXCR4. Furthermore, miR-150 has been shown to regulate cerebral angiogenesis through down-regulation of VEGF in a rat model of middle cerebral artery occlusion. ${ }^{11}$ However, a functional involvement of miR-150 in the prognosis after stroke in the present study remains at a speculative level and deserves further investigation.

In humans, the potential protective role of miR-150-5p has also been studied in various other diseases where endothelial injury plays a key role. Higher levels of miR-150-5p were associated with a lower mortality rate in patients with acute sep$\mathrm{sis}_{1}{ }^{26}$ critically ill patients, ${ }_{1}^{27}$ pulmonary arterial hypertension ${ }^{28}$ as well as in myocardial infarction. ${ }^{8}$ In a recent study a polymorphism in the gene encoding for miR-150 was found to be associated with ischemic stroke. ${ }^{29}$ Additionally, Cox proportional analysis indicated that the miR-150GA genotype was associated with survival in patients with ischemic stroke. ${ }^{29}$

The available basic and clinical data are in line with our observation that log-miR-150-5p was associated with a reduced mortality after ischemic stroke. However, we did not find a significant association with functional outcome. A potential explanation for this observation would be that higher log-miR150-5p levels reduce risk of systemic secondary complications after stroke, such as myocardial infarction or pneumonia, which are associated with higher mortality in this population. However, log-miR-150-5p levels may not directly improve for example brain plasticity thus explaining the lack of a direct association with functional outcome. Alternatively, the protective role underlies a threshold effect and miRNA levels below a certain value reflect a point of no return rather than a gradual association with improved functional outcome. The exact mechanisms of the factors that regulate the change of log-miR-150$5 p$ expression after stroke remain to be fully elucidated.

Some limitations of our study merit attention. First, plasma samples were stored at $-70^{\circ} \mathrm{C}$ for several years before measurement of miR-150-5p, which could potentially lead to some degradation. However, degradation would similarly have affected survivors and non-survivors, and it is commonly accepted that miRNAs are stable for several years during $-70^{\circ} \mathrm{C}$ storage. Pre-analytic problems can always affect measurements but we only found signs (i.e., change of colour) of potential hemolysis in 8\% of all miR-150-5p measurements in our cohort. To assess a potential effect modification, we conducted multivariate analysis (Table 3) without and with these samples respectively and didn't detected any significant changes with
(HR 0.21 [95\% $\mathrm{Cl}, 0.08-0.51], P=0.001$ ) and without hemolysis (HR 0.22 [95\% Cl, 0.08-0.61], $P=0.004$ ). Moreover, also hemolysis would similarly have affected survivors and non-survivors.

Second, due to some missing baseline values, we used imputation methods in order to reduce potential bias and retain a sufficient level of statistical power. Noteworthy, the direction, magnitude as well as significance level of the association of log-miR-150-5p with mortality were very similar with and without imputation ( $\mathrm{HR} 0.21$ [95\% $\mathrm{Cl}, 0.08-0.51]$ and $\mathrm{HR} 0.17$ [95\% Cl, 0.06-0.48], respectively). Furthermore, imputation did not affect the value of log-miR-150-5p to discriminate those who died compared to those who survived (AUC $0.92[95 \% \mathrm{Cl}$, 0.88-0.96] with and 0.91 [95\% Cl, 0.86-0.96] without imputation, respectively), this suggests that the results are reliable. Third, repeated measurements of log-miR-150-5p or their changes over time may be a better indicator of mortality than levels measured at a single baseline point. However, such kinetic data are currently unavailable. A last limitation is the selection of one candidate miRNA to be included in these analyses, which does not preclude that other miRNAs may have a prognostic value after ischemic stroke as well.

Third, developments of new therapeutic approaches in stroke could affect the results of this study too.

Fourth, our NRI could be false positive. For this reason we conducted additionally a pseudo R-squared test which strengthen our results. Further independent validation cohort studies are necessary.

The strengths of this study include the systematic and preplanned collection of clinical data, the highly standardized acquisition and processing of blood within a clear time window and the ability to adjust for numerous covariates. Moreover, we could assess the prognostic value also in a subgroup of patients with measured diffusion-weighted imaging lesions and found that $\log -\mathrm{miR}-150-5 p$ was still associated with mortality independently of lesion size. Finally, we performed several analyses to address a potential incremental value of log-miR-150-5p beyond established demographic and vascular risk factors, which all pointed in the same direction, i.e., a gain of prediction accuracy by log-miR-150-5p.

Our findings could have potential clinical implications. LogmiR-150-5p might help in early decision making concerning intensive care monitoring, discharge and rehabilitation planning. In the setting of trials of new stroke therapies, log-miR150-5p could be of value in selecting those, which may benefit most of an intervention. For optimal risk stratification, it is crucial that prognostic information is available within the first hours from symptom onset and that it adds prognostic information beyond known established factors, and log-miR-150- 
$5 p$ meets this demand. However, before considering the use of $\log -m i R-150-5 p$ in clinical practice, the prognostic value of log-miR-150-5p needs to be validated in prospective, independent, large, multicenter studies according to the recommendations of the American Heart Association for studies evaluating biomarkers in cardiovascular research. ${ }^{30}$

\section{Conclusions}

In conclusion, our results suggest that log-miR-150-5p is a novel prognostic biomarker, improving the risk classification for mortality in patients with acute ischemic stroke independently from traditional demographic, vascular and imaging risk factors. Further studies are required to validate this finding.

\section{Supplementary Materials}

Supplementary materials related to this article can be found online at https://doi.org/10.5853/jos.2017.00423.

\section{References}

1. Ntaios G, Faouzi M, Ferrari J, Lang W, Vemmos K, Michel P. An integer-based score to predict functional outcome in acute ischemic stroke: the ASTRAL score. Neurology 2012; 78:1916-1922.

2. Saposnik G, Kapral MK, Liu Y, Hall R, O'Donnell M, Raptis $S$, et al. IScore: a risk score to predict death early after hospitalization for an acute ischemic stroke. Circulation 2011;123: 739-749.

3. Whiteley W, Tseng MC, Sandercock P. Blood biomarkers in the diagnosis of ischemic stroke: a systematic review. Stroke 2008:39:2902-2909.

4. Whiteley W, Chong WL, Sengupta A, Sandercock P. Blood markers for the prognosis of ischemic stroke: a systematic review. Stroke 2009;40:e380-e389.

5. De Marchis GM, Katan M, Weck A, Fluri F, Foerch C, Findling 0 , et al. Copeptin adds prognostic information after ischemic stroke: results from the CoRisk study. Neurology 2013;80: 1278-1286.

6. Salic K, De Windt $\amalg$. MicroRNAs as biomarkers for myocardial infarction. Curr Atheroscler Rep 2012;14:193-200.

7. Gilad $S_{1}$ Meiri $E_{1}$ Yogev $Y$, Benjamin $S$, Lebanony $D$, Yerushalmi N, et al. Serum microRNAs are promising novel biomarkers. PLoS One 2008;3:e3148.

8. Devaux Y, Vausort M, McCann GP, Zangrando J, Kelly D, Razvi N, et al. MicroRNA-150: a novel marker of left ventricular remodeling after acute myocardial infarction. Circ Cardiovasc
Genet 2013;6:290-298.

9. Liu Z, Ye P, Wang $S$, Wu J, Sun Y, Zhang A, et al. MicroRNA-150 protects the heart from injury by inhibiting monocyte accumulation in a mouse model of acute myocardial infarction. Circ Cardiovasc Genet 2015;8:11-20.

10. Zhang Y, Liu D, Chen X, Li J, Li L, Bian Z, et al. Secreted monocytic miR-150 enhances targeted endothelial cell migration. Mol Cell 2010;39:133-144.

11. He OW, Li Q, Jin HJ, Zhi F, Suraj B, Zhu YY, et al. MiR-150 regulates poststroke cerebral angiogenesis via vascular endothelial growth factor in rats. CNS Neurosci Ther 2016;22: 507-517.

12. Katan $M$, Fluri F, Morgenthaler NG, Schuetz $P$, Zweifel $C$, Bingisser $\mathrm{R}$, et al. Copeptin: a novel, independent prognostic marker in patients with ischemic stroke. Ann Neurol 2009; 66:799-808

13. Waltimo $\mathrm{O}$, Kaste $\mathrm{M}$, Aho $\mathrm{K}$, Kotila M. Outcome of stroke in the Espoo--Kauniainen area, Finland. Ann Clin Res 1980;12: 326-330.

14. Brott $T$, Adams HP Jr, Olinger CP, Marler JR, Barsan WG, Biller $J$, et al. Measurements of acute cerebral infarction: a clinical examination scale. Stroke 1989:20:864-870.

15. Adams HP Jr, Bendixen BH, Kappelle ப, Biller J, Love BB, Gordon DL, et al. Classification of subtype of acute ischemic stroke. Definitions for use in a multicenter clinical trial. TOAST. Trial of Org 10172 in Acute Stroke Treatment. Stroke 1993;24:35-41.

16. Devaux $Y$, Vausort $M$, Goretti E, Nazarov PV, Azuaje F, Gilson $G$, et al. Use of circulating microRNAs to diagnose acute myocardial infarction. Clin Chem 2012;58:559-567.

17. Devaux Y, Dankiewicz J, Salgado-Somoza A, Stammet P, Collignon 0 , Gilje $P$, et al. Association of circulating microRNA124-3p levels with outcomes after out-of-hospital cardiac arrest: a substudy of a randomized clinical trial. JAMA Cardiol 2016;1:305-313.

18. Sims JR, Gharai LR, Schaefer PW, Vangel M, Rosenthal ES, Lev $M H$, et al. $A B C / 2$ for rapid clinical estimate of infarct, perfusion, and mismatch volumes. Neurology 2009;72:21042110.

19. Li P, Stuart EA, Allison DB. Multiple imputation: a flexible tool for handling missing data. JAMA 2015;314:1966-1967.

20. White IR, Royston P, Wood AM. Multiple imputation using chained equations: issues and guidance for practice. Stat Med 2011;30:377-399.

21. Pencina MJ, D'Agostino RB Sr, Steyerberg EW. Extensions of net reclassification improvement calculations to measure usefulness of new biomarkers. Stat Med 2011;30:11-21.

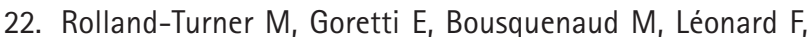


Nicolas C, Zhang L, et al. Adenosine stimulates the migration of human endothelial progenitor cells. Role of CXCR4 and microRNA-150. PLoS One 2013;8:e54135.

23. Goren $Y$, Meiri $E_{1}$ Hogan $C$, Mitchell $H$, Lebanony D, Salman $\mathrm{N}$, et al. Relation of reduced expression of MiR-150 in platelets to atrial fibrillation in patients with chronic systolic heart failure. Am J Cardiol 2014;113:976-981.

24. Fleissner F, Jazbutyte V, Fiedler J, Gupta SK, Yin X, Xu Q, et al. Short communication: asymmetric dimethylarginine impairs angiogenic progenitor cell function in patients with coronary artery disease through a microRNA-21-dependent mechanism. Circ Res 2010;107:138-143.

25. Xiao C, Calado DP, Galler G, Thai TH, Patterson HC, Wang J, et al. MiR-150 controls $B$ cell differentiation by targeting the transcription factor c-Myb. Cell 2007;131:146-159.

26. Vasilescu C, Rossi S, Shimizu M, Tudor S, Veronese A, Ferracin $M$, et al. MicroRNA fingerprints identify miR-150 as a plasma prognostic marker in patients with sepsis. PLoS One 2009;4:e7405.
27. Roderburg C, Luedde M, Vargas Cardenas D, Vucur M, Scholten $D$, Frey $N$, et al. Circulating microRNA-150 serum levels predict survival in patients with critical illness and sepsis. PLoS One 2013;8:e54612.

28. Rhodes CJ, Wharton J, Boon RA, Roexe T, Tsang H, WojciakStothard $B$, et al. Reduced microRNA-150 is associated with poor survival in pulmonary arterial hypertension. Am J Respir Crit Care Med 2013;187:294-302.

29. Choi GH, Ko KH, Kim JO, Kim J, Oh SH, Han IB, et al. Association of miR-34a, miR-130a, miR-150 and miR-155 polymorphisms with the risk of ischemic stroke. Int J Mol Med 2016; 38:345-356.

30. Hlatky MA, Greenland P, Arnett DK, Ballantyne CM, Criqui $\mathrm{MH}$, Elkind MS, et al. Criteria for evaluation of novel markers of cardiovascular risk: a scientific statement from the American Heart Association. Circulation 2009;119:2408-2416.

31. Vickers AJ, Cronin AM, Begg CB. One statistical test is sufficient for assessing new predictive markers. BMC Med Res Methodol 2011;11:13. 

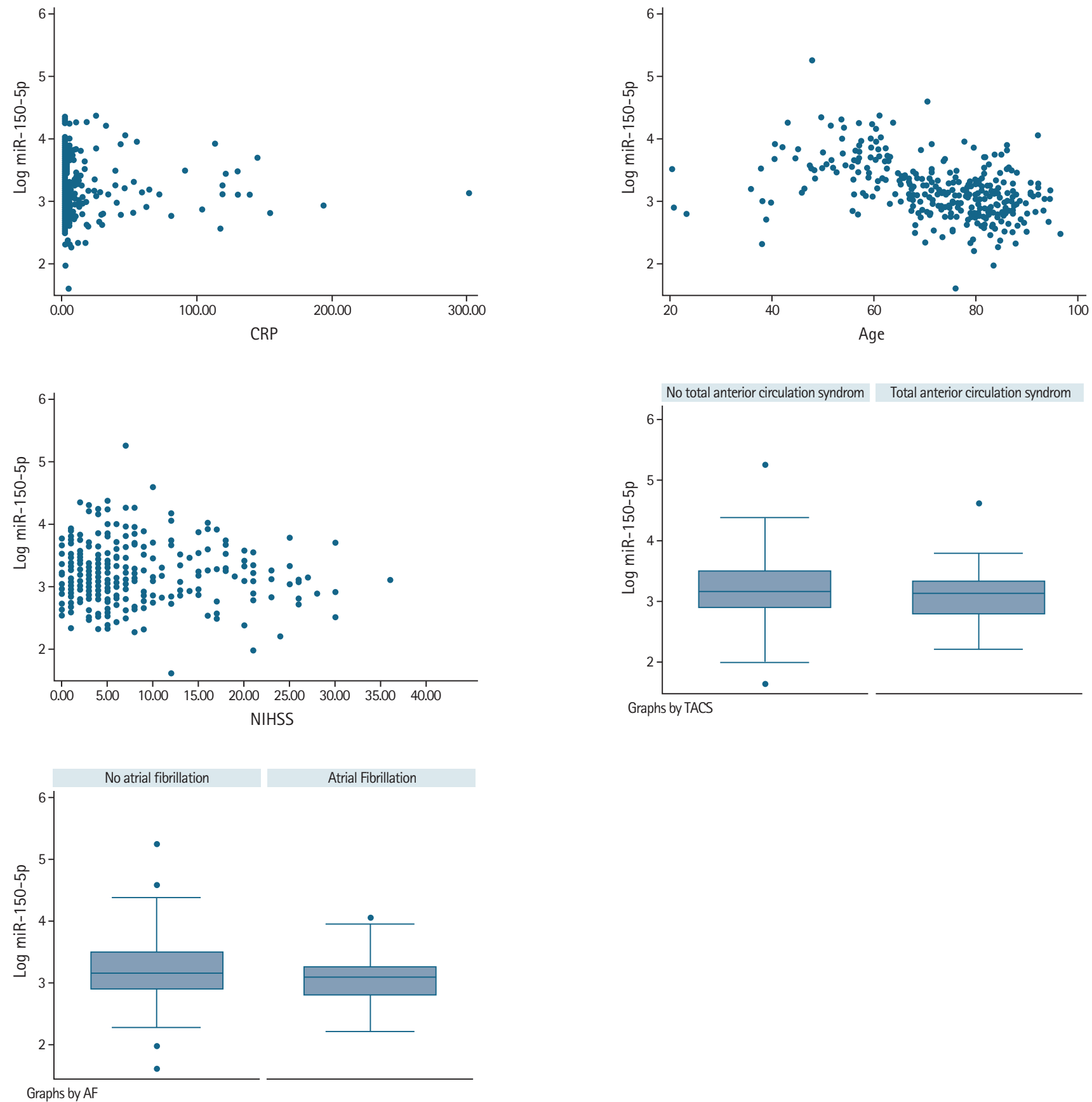

Supplementary Figure 1. These figures show the scatter and box plot of the log-miR150 and the risk factors, which were statistically significant in the univariate analyses for mortality. CRP, C-reactive protein; NIHSS, National Institute of Health Stroke Scale; TACS, total anterior circulation syndrome; AF, atrial fibrillation. 